\title{
A Variational Approach to Perturbed Discrete Anisotropic Equations
}

\author{
Amjad Salari, ${ }^{1}$ Giuseppe Caristi, ${ }^{2}$ David Barilla, ${ }^{2}$ and Alfio Puglisi ${ }^{2}$ \\ ${ }^{1}$ Department of Mathematics, Faculty of Sciences, Razi University, Kermanshah 67149, Iran \\ ${ }^{2}$ Department of Economics, University of Messina, Via dei Verdi 75, Messina, Italy \\ Correspondence should be addressed to Giuseppe Caristi; gcaristi@unime.it
}

Received 2 July 2016; Accepted 10 October 2016

Academic Editor: Patricia J. Y. Wong

Copyright (c) 2016 Amjad Salari et al. This is an open access article distributed under the Creative Commons Attribution License, which permits unrestricted use, distribution, and reproduction in any medium, provided the original work is properly cited.

\begin{abstract}
We continue the study of discrete anisotropic equations and we will provide new multiplicity results of the solutions for a discrete anisotropic equation. We investigate the existence of infinitely many solutions for a perturbed discrete anisotropic boundary value problem. The approach is based on variational methods and critical point theory.
\end{abstract}

\section{Introduction}

The aim of this paper is to investigate the existence of infinitely many solutions for the following perturbed discrete anisotropic problem:

$$
\begin{aligned}
& -\Delta\left(\alpha(k)|\Delta u(k-1)|^{p(k-1)-2} \Delta u(k-1)\right) \\
& \quad=\lambda f(k, u(k))+\mu g(k, u(k)), \quad k \in[1, T], \quad\left(P_{\lambda}^{f, g}\right) \\
& u(0)=u(T+1)=0,
\end{aligned}
$$

where $T \geq 2$ is a fixed positive integer number, $[1, T]$ is the discrete interval $\{1, \ldots, T\} \subset \mathbb{N}, \Delta u(k-1)=u(k)-u(k-1)$, $\alpha:[1, T+1] \rightarrow(0,+\infty)$, and $p:[0, T] \rightarrow(1,+\infty)$ are some fixed functions, and $f, g:[1, T] \times \mathbb{R} \rightarrow \mathbb{R}$ are two continuous functions. Let

$$
\begin{aligned}
& p^{-}=\min _{k \in[0, T]} p(k), \\
& p^{+}=\max _{k \in[0, T]} p(k), \\
& \alpha^{-}=\min _{k \in[1, T+1]} \alpha(k), \\
& \alpha^{+}=\max _{k \in[1, T+1]} \alpha(k) .
\end{aligned}
$$

Many problems in applied mathematics lead to the study of discrete boundary value problems and difference equations. Indeed, common among many fields of research, such as computer science, mechanical engineering, control systems, artificial or biological neural networks, and economics, is the fact that the mathematical modelling of fundamental questions is usually tended towards considering discrete boundary value problems and nonlinear difference equations. Regarding these issues, a thoroughgoing overview has been given in, as an example, the monograph [1] and the reference therein. On the other hand, in recent years some researchers have studied the existence and multiplicity of solutions for equations involving the discrete $p$-Laplacian operator by using various fixed point theorems, lower and upper solutions method, critical point theory and variational methods, Morse theory, and the mountain-pass theorem. For background and recent results, we refer the reader to [2-20] and the references therein. For example, Atici and Guseinov in [3] investigated the existence of positive periodic solutions for nonlinear difference equations with periodic coefficients by employing a fixed point theorem in cone. Atici and Cabada in [2], by using the upper and lower solution method, obtained the existence and uniqueness results for a discrete boundary value problem. Henderson and Thompson in [12] gave conditions on the nonlinear term involving pairs of discrete lower and discrete upper solutions which led to the existence of at least three solutions of discrete two-point boundary value problems, and in a special case of the nonlinear term they gave growth conditions on the function and applied their general result to show the existence of three positive solutions. Chu and 
Jiang in [9] based on fixed point theorem in a cone, due to Krasnoselskii, characterized the eigenvalues and showed the existence of positive solutions to a discrete boundary value problem. Jiang and Zhou in [13] employing a three-critical point theorem, due to Ricceri, established the existence of at least three solutions for perturbed nonlinear difference equations with discrete boundary conditions. Wang and Guan in [20], by using the Five Functionals Fixed Point Theorem, obtained the existence criteria for three positive solutions of a $p$-Laplacian difference equation. Bonanno and Candito [5], employing critical point theorems in the setting of finite dimensional Banach spaces, investigated the multiplicity of solutions for nonlinear difference equations involving the $p$-Laplacian. Cabada et al. in [6], based on three critical points' theorems, investigated different sets of assumptions which guarantee the existence and multiplicity of solutions for difference equations involving the discrete $p$-Laplacian operator. In [4] Bian et al. by using critical point theory studied a class of discrete $p$-Laplacian periodic boundary value problems; some results were obtained for the existence of two positive solutions, three solutions, and multiple pairs of solutions of the problem when the parameter lies in some suitable infinite or finite intervals. In [15], by using variational methods and critical point theory, the existence of infinitely many solutions for perturbed nonlinear difference equations with discrete Dirichlet boundary conditions was discussed.

There seems to be increasing interest in the existence of solutions to discrete anisotropic equations, because of their applications in many fields such as models in physics [21$24]$, biology [25, 26], and image processing (see, for example, Weickert's monograph [27]). We also mention Fragalà et al. [28] and El Hamidi and Vétois [29] as essential references in treating the nonlinear anisotropic problems. Besides, Mihăilescu et al. (see $[30,31]$ ) were the first authors who studied anisotropic elliptic problems with variable exponents. On the other hand, numerous researches have been undertaken on the existence of solutions for discrete anisotropic boundary value problems (BVPs) in recent years. As to the background and latest results, the readers can refer to [32$38]$ and the references therein. For example, Mihăilescu et al. in [36], by using critical point theory, obtained the existence of a continuous spectrum for a family of discrete boundary value problems. Galewski and Wieteska in [34] investigated the existence of solutions of the system of anisotropic discrete boundary value problems using critical point theory, while in [33], using variational methods, they derived the intervals of the numerical parameter for which the problem $\left(P_{\lambda}^{f, g}\right)$ has at least 1 , exactly 1 , or at least 2 positive solutions. They also derived some useful discrete inequalities. Molica Bisci and Repovš in [37] considered advantage of a recent critical point theorem to establish the existence of infinitely many solutions for anisotropic difference equation:

$$
\begin{aligned}
&-\Delta\left(|\Delta u(k-1)|^{p(k-1)-2} \Delta u(k-1)\right)= \lambda f_{k}(u(k)), \\
& k \in \mathbb{Z}[1, T], \\
& u(0)=u(T+1)=0,
\end{aligned}
$$

where $\lambda$ is a positive parameter, $f_{k}: \mathbb{R} \rightarrow \mathbb{R}$ is a continuous function for every $k \in \mathbb{Z}[1, T]$ (with $T \geq 2$ ), and $\Delta u(k-1):=$ $u(k)-u(k-1)$ is the forward difference operator, assuming that the map $p: \mathbb{Z}[0, T] \rightarrow \mathbb{R}$ satisfies $p^{-}:=\min _{\mathbb{Z}[0, T]} p(k)>$ 1 as well as $p^{+}:=\max _{\mathbb{Z}[0, T]} p(k)>1$. Stegliński in [38], based on critical point theory, obtained the existence of infinitely many solutions for the parametric version of the problem $\left(P_{\lambda}^{f, g}\right)$, in the case where $\mu=0$.

Motivated by the above works, in the present paper, by employing a smooth version of [39, Theorem 2.1], which is more precise version of Ricceri's Variational Principle [40, Theorem 2.5] under some hypotheses on the behavior of the nonlinear terms at infinity, we prove the existence of definite intervals about $\lambda$ and $\mu$ in which the problem $\left(P_{\lambda}^{f, g}\right)$ admits a sequence of solutions which is unbounded in the space $E$ which will be introduced later (Theorem 6). Furthermore, some consequences of Theorem 6 are listed. A partial case of main result is formulated as Theorem 5. Replacing the conditions at infinity on the nonlinear terms, by a similar one at zero, we obtain a sequence of pairwise distinct solutions strongly converging at zero; see Theorem 14 . Three examples of applications are pointed out (see Examples 8, 13, and 16).

\section{Preliminaries}

Our main tool to ensure the existence of infinitely many solutions for the problem $\left(P_{\lambda}^{f, g}\right)$ is a smooth version of Theorem 2.1 of [39] which is a more precise version of Ricceri's Variational Principle [40] that we now recall here.

Theorem 1. Let $X$ be a reflexive real Banach space and let $\Phi, \Psi: X \rightarrow \mathbb{R}$ be two Gâteaux differentiable functionals such that $\Phi$ is sequentially weakly lower semicontinuous, strongly continuous, and coercive and $\Psi$ is sequentially weakly upper semicontinuous. For every $r>\inf _{X} \Phi$, let one put

$$
\begin{aligned}
\varphi(r) & :=\inf _{u \in \Phi^{-1}(]-\infty, r[)} \frac{\sup _{\left.\left.v \in \Phi^{-1}(]-\infty, r\right]\right)} \Psi(v)-\Psi(u)}{r-\Phi(u)}, \\
\gamma & :=\liminf _{r \rightarrow+\infty} \varphi(r), \\
\delta & :=\liminf _{\left.r \rightarrow \inf _{X} \Phi\right)^{+}} \varphi(r) .
\end{aligned}
$$

Then, one has the following:

(a) For every $r>\inf _{X} \Phi$ and every $\left.\lambda \in\right] 0,1 / \varphi(r)[$, the restriction of the functional $I_{\lambda}=\Phi-\lambda \Psi$ to $\Phi^{-1}(]-$ $\infty, r[)$ admits a global minimum, which is a critical point (local minimum) of $I_{\lambda}$ in $X$.

(b) If $\gamma<+\infty$, then, for each $\lambda \in] 0,1 / \gamma$ [, the following alternative holds:

either

\section{$\left(b_{1}\right) I_{\lambda}$ possesses a global minimum}

or

$\left(b_{2}\right)$ there is a sequence $\left\{u_{n}\right\}$ of critical points (local minima) of $I_{\lambda}$ such that

$$
\lim _{n \rightarrow+\infty} \Phi\left(u_{n}\right)=+\infty \text {. }
$$


(c) If $\delta<+\infty$, then, for each $\lambda \in] 0,1 / \delta[$, the following alternative holds:

either

$\left(c_{1}\right)$ there is a global minimum of $\Phi$ which is a local minimum of $I_{\lambda}$

or

$\left(c_{2}\right)$ there is a sequence of pairwise distinct critical points (local minima) of $I_{\lambda}$ which weakly converges to a global minimum of $\Phi$.

We refer the reader to the paper [41-47] in which Theorem 1 was successfully employed to ensure the existence of infinitely many solutions for boundary value problems.

Here and in the sequel we take the $T$-dimensional Banach space

$$
E:=\{u:[0, T+1] \longrightarrow \mathbb{R}: u(0)=u(T+1)=0\},
$$

endowed with the norm

$$
\|u\|:=\left(\sum_{k=1}^{T+1}|\Delta u(k-1)|^{2}\right)^{1 / 2} .
$$

Remark 2. We consider that whenever $E$ is a finite dimensional Banach space in Theorem 1, in order to show the regularity of the derivative of $\Phi$ and $\Psi$, it is merely enough to indicate that $\Phi^{\prime}$ and $\Psi^{\prime}$ are two continuous functionals on $E^{*}$.

Lemma 3 (see [48, Section 2]). (a.1) For every $u \in E$ with $\|u\|>1$ we have

$$
\sum_{k=1}^{T+1}|\Delta u(k-1)|^{p(k-1)} \geq T^{\left(2-p^{-}\right) / 2}\|u\|^{p^{-}}-T .
$$

(a.2) For every $u \in E$ with $\|u\| \leq 1$ we have

$$
\sum_{k=1}^{T+1}|\Delta u(k-1)|^{p(k-1)} \geq T^{\left(p^{+}-2\right) / 2}\|u\|^{p^{+}} .
$$

(a.3) For every $u \in E$ and for any $m \geq 2$ we have

$$
\begin{aligned}
(T+1)^{(2-m) / 2}\|u\|^{m} & \leq \sum_{k=1}^{T+1}|\Delta u(k-1)|^{m} \\
& \leq(T+1)\|u\|^{m}
\end{aligned}
$$

(a.4) If $p^{+} \geq 2$ there exists positive constant $C_{p^{+}}$such that for every $u \in E$

$$
\sum_{k=1}^{T+1}|\Delta u(k-1)|^{p(k-1)} \leq 2^{p^{+}}(T+1)\left(C_{p^{+}}\|u\|^{p^{+}}+1\right) .
$$

(a.5) For every $u \in E$ and for any $m \geq 2$ we have

$$
\sum_{k=1}^{T+1}|\Delta u(k-1)|^{m} \leq 2^{m} \sum_{k=1}^{T}|u(k)|^{m} .
$$

(a.6) For every $u \in E$ and for any $p, q>1$ such that $1 / p+$ $1 / q=1$ we have

$$
\begin{aligned}
\|u\|_{\infty} & =\max _{k \in[1, T]}|u(k)| \\
& \leq(T+1)^{1 / q}\left(\sum_{k=1}^{T+1}|\Delta u(k-1)|^{p}\right)^{1 / p} .
\end{aligned}
$$

Put

$$
F(k, t):=\int_{0}^{t} f(k, \xi) \mathrm{d} \xi \quad \forall(k, t) \in[1, T] \times \mathbb{R} .
$$

Remark 4. We recall that a map $f:[1, T] \times \mathbb{R} \rightarrow \mathbb{R}$ is continuous if it is continuous as a map of the topological space $[1, T] \times \mathbb{R}$ into the topological space $\mathbb{R}$. In this paper, the topology on $[1, T]$ will be the discrete topology.

A special case of our main result is the following theorem.

Theorem 5. Let $f: \mathbb{R} \rightarrow \mathbb{R}$ be a continuous function and put $F(t)=\int_{0}^{t} f(\xi) \mathrm{d} \xi$ for all $t \in \mathbb{R}$. Assume that

$$
\begin{aligned}
\liminf _{\xi \rightarrow+\infty} \frac{F(\xi)}{\xi^{p}} & =0, \\
\limsup _{\xi \rightarrow+\infty} \frac{F(\xi)}{\xi^{p}} & =+\infty .
\end{aligned}
$$

Then, for every continuous function $g: \mathbb{R} \rightarrow \mathbb{R}$ whose $G(t)=$ $\int_{0}^{t} g(\xi) \mathrm{d} \xi$, for every $t \in \mathbb{R}$, is a nonnegative function satisfying the condition

$$
g_{\star}:=T^{p} p \lim _{\xi \rightarrow+\infty} \frac{\sup _{|t| \leq \xi} G(t)}{\xi^{p}}<+\infty
$$

and for every $\mu \in\left[0, \mu_{\star, \lambda}\left[\right.\right.$, where $\mu_{\star, \lambda}:=\left(1 / g_{\star}\right)(1-$ $\left.\lambda T^{p} p \lim \inf _{\xi \rightarrow+\infty}\left(F(\xi) / \xi^{p}\right)\right)$, the problem

$$
\begin{aligned}
& -\Delta\left(|\Delta u(k-1)|^{p-2} \Delta u(k-1)\right) \\
& \quad=\lambda f(u(k))+\mu g(u(k)), \quad k \in[1, T], \\
& u(0)=u(T+1)=0
\end{aligned}
$$

has an unbounded sequence of solutions.

\section{Main Results}

We present our main result as follows.

Theorem 6. Assume that

(A1) $\liminf \operatorname{in\rightarrow +\infty }_{\xi \rightarrow}\left(\sum_{k=1}^{T} \sup _{|t| \leq \xi} F(k, t) / \xi^{p^{-}}\right)<\left(p^{-} \alpha^{-} /\right.$ $\left.2 \alpha^{+} T^{p^{-}} p^{+}\right) \lim \sup _{\xi \rightarrow+\infty}\left(\sum_{k=1}^{T} F(k, \xi) / \xi^{p^{+}}\right)$.

Then, for each $\lambda \in] \lambda_{1}, \lambda_{2}[$, where

$$
\begin{aligned}
& \lambda_{1}:=\frac{2 \alpha^{+}}{p^{-} \lim \sup _{\xi \rightarrow+\infty}\left(\sum_{k=1}^{T} F(t, \xi) / \xi p^{+}\right)}, \\
& \lambda_{2}:=\frac{\alpha^{-}}{T p^{-} p^{+} \liminf _{\xi \rightarrow+\infty}\left(\sum_{k=1}^{T} \sup _{|t| \leq \xi} F(k, t) / \xi p^{-}\right)},
\end{aligned}
$$


for every continuous function $g:[1, T] \times \mathbb{R} \rightarrow \mathbb{R}$ whose $G(k, t)=\int_{0}^{t} g(k, \xi) \mathrm{d} \xi$ for every $(k, t) \in[1, T] \times \mathbb{R}$, is a nonnegative function satisfying the condition

$$
g_{\infty}:=\frac{T^{p^{-}} p^{+}}{\alpha^{-}} \lim _{\xi \rightarrow+\infty} \frac{\sum_{k=1}^{T} \sup _{|t| \leq \xi} G(k, t)}{\xi^{p^{-}}}<\infty
$$

and for every $\mu \in\left[0, \mu_{g, \lambda}[\right.$, where

$$
\begin{aligned}
& \mu_{g, \lambda} \\
& \quad:=\frac{1}{g_{\infty}}\left(1-\frac{\lambda T^{p^{-}} p^{+}}{\alpha^{-}} \liminf _{\xi \rightarrow+\infty} \frac{\sum_{k=1}^{T} \sup _{|t| \leq \xi} F(k, t)}{\xi^{-}}\right),
\end{aligned}
$$

the problem $\left(P_{\lambda}^{f, g}\right)$ has an unbounded sequence of solutions.

Proof. Fix $\bar{\lambda} \in] \lambda_{1}, \lambda_{2}$ [ and let $g$ be a function satisfying the condition (18). Since $\bar{\lambda}<\lambda_{2}$, one has $\mu_{g, \bar{\lambda}}>0$. Fix $\bar{\mu} \in\left[0, \mu_{g, \bar{\lambda}}[\right.$ and put $\nu_{1}:=\lambda_{1}$ and $\nu_{2}:=\lambda_{2} /\left(1+(\bar{\mu} / \bar{\lambda}) \lambda_{2} g_{\infty}\right)$. If $g_{\infty}=$ 0 , clearly, $v_{1}=\lambda_{1}, v_{2}=\lambda_{2}$, and $\left.\bar{\lambda} \in\right] v_{1}, v_{2}$ [. If $g_{\infty} \neq 0$, since $\bar{\mu}<\mu_{g, \bar{\lambda}}$, we obtain $\bar{\lambda} / \lambda_{2}+\bar{\mu} g_{\infty}<1$, and so $\lambda_{2} /(1+$ $\left.(\bar{\mu} / \bar{\lambda}) \lambda_{2} g_{\infty}\right)>\bar{\lambda}$, namely, $\bar{\lambda}<v_{2}$. Hence, since $\bar{\lambda}>\lambda_{1}=v_{1}$, one has $\bar{\lambda} \epsilon] \nu_{1}, \nu_{2}[$. Now, set $Q(k, t)=F(k, t)+(\bar{\mu} / \bar{\lambda}) G(k, t)$ for all $(k, t) \in[1, T] \times \mathbb{R}$. Take $X=E$ and define on $X$ two functionals $\Phi$ and $\Psi$ as follows:

$$
\begin{aligned}
& \Phi(u)=\sum_{k=1}^{T+1} \frac{\alpha(k)}{p(k-1)}|\Delta u(k-1)|^{p(k-1)}, \\
& \Psi(u)=\sum_{k=1}^{T} F(k, u(k))+\frac{\bar{\mu}}{\bar{\lambda}} \sum_{k=1}^{T} G(k, u(k)) .
\end{aligned}
$$

Since $X$ is a finite dimensional Banach space, $\Psi$ is a Gâteaux differentiable functional and sequentially weakly upper semicontinuous whose Gâteaux derivative at the point $u \in X$ is the functional $\Psi^{\prime}(u) \in X^{*}$, given by

$$
\begin{aligned}
\Psi^{\prime}(u) v= & \sum_{k=1}^{T} f(k, u(k)) v(k) \\
& +\frac{\bar{\mu}}{\bar{\lambda}} \sum_{k=1}^{T} g(k, u(k)) v(k),
\end{aligned}
$$

for every $v \in X$, and $\Psi^{\prime}: X \rightarrow X^{*}$ is a compact operator. Moreover, $\Phi$ is a Gâteaux differentiable functional of which Gâteaux derivative at the point $u \in X$ is the functional $\Phi^{\prime}(u) \in X^{*}$, given by

$$
\begin{aligned}
\Phi^{\prime} & (u)(v) \\
= & \sum_{k=1}^{T+1} \alpha(k)|\Delta u(k-1)|^{p(k-1)-2} \Delta u(k-1) \Delta v(k-1)
\end{aligned}
$$

for every $v \in X$. Furthermore, $\Phi$ is sequentially weakly lower semicontinuous (see [5, Remarks 2.2 and 2.3]). Put $I_{\bar{\lambda}}:=\Phi-$ $\bar{\lambda} \Psi$. We observe that the solutions of the problem $\left(P_{\lambda}^{f, g}\right)$ are exactly the solutions of the equation $I_{\bar{\lambda}}^{\prime}(u)=0$. So, our end is to apply Theorem 1 to $\Phi$ and $\Psi$. Now, we wish to prove that $\gamma<+\infty$, where $\gamma$ is defined in Theorem 1. Let $\left\{\xi_{n}\right\}$ be a real sequence such that $\xi_{n}>0$ for all $n \in \mathbb{N}$ and $\xi_{n} \rightarrow+\infty$ as $n \rightarrow \infty$ and

$$
\begin{aligned}
\lim _{n \rightarrow \infty} \frac{\sum_{k=1}^{T} \sup _{|t| \leq \xi_{n}} Q(k, t)}{\xi_{n}^{p^{-}}} \\
=\liminf _{\xi \rightarrow+\infty} \frac{\sum_{k=1}^{T} \sup _{|t| \leq \xi} Q(k, t)}{\xi^{p^{-}}} .
\end{aligned}
$$

Put $r_{n}=\left(\alpha^{-} / T^{p^{-}} p^{+}\right) \xi_{n}^{p^{-}}$for all $n \in \mathbb{N}$. Let $n_{0} \in \mathbb{N}$ be such that $\left(p^{+} / \alpha^{-}\right) r_{n}>1$ for all $n>n_{0}$. We claim that

$$
\Phi^{-1}\left(\left(-\infty, r_{n}\right)\right) \subseteq\left\{u:\|u\|_{\infty} \leq \xi_{n} \forall k \in[0, T+1]\right\} .
$$

Indeed, if $v \in X$ and $\phi(v)<r_{m}$, one has

$$
\sum_{k=1}^{T+1} \frac{\alpha(k)}{p(k-1)}|\Delta u(k-1)|^{p(k-1)}<r_{m} .
$$

Then,

$$
|\Delta u(k-1)|<\left(\frac{p(k-1)}{\alpha(k)} r_{m}\right)^{1 / p(k-1)} \leq\left(\frac{p^{+}}{\alpha^{-}} r_{m}\right)^{1 / p^{-}}
$$

for every $k \in[1, T+1]$. Consequently, since $u \in X$, we deduce by easy induction that

$$
\begin{aligned}
|u(k)| & \leq|\Delta u(k-1)|+|u(k-1)| \\
& <\left(\frac{p^{+}}{\alpha^{-}} r_{n}\right)^{1 / p^{-}}+|u(k-1)| \leq k\left(\frac{p^{+}}{\alpha^{-}} r_{n}\right)^{1 / p^{-}} \\
& \leq T\left(\frac{p^{+}}{\alpha^{-}} r_{n}\right)^{1 / p^{-}}=\xi_{n}
\end{aligned}
$$

for every $k \in[1, T]$ and this gives (24). Hence, taking into account the fact that $\Phi(0)=\Psi(0)=0$, for every $n$ large enough, one has

$$
\begin{aligned}
& \varphi\left(r_{n}\right)=\inf _{u \in \Phi^{-1}(]-\infty, r_{n}[)} \frac{\left(\sup _{\left.\left.v \in \Phi^{-1}(]-\infty, r_{n}\right]\right)} \Psi(v)\right)-\Psi(u)}{r_{n}-\Phi(u)} \\
& \leq \frac{\sup _{\left.\left.v \in \Phi^{-1}(]-\infty, r_{n}\right]\right)} \Psi(v)}{r_{n}} \\
& \leq \frac{\sum_{k=1}^{T} \sup _{|t| \leq \xi_{n}} Q(k, t)}{\alpha^{-} \xi_{n}^{p^{-}} / T^{p^{-}} p^{+}} \\
& =\frac{\sum_{k=1}^{T} \sup _{|t| \leq \xi_{n}} F(k, t)+(\bar{\mu} / \bar{\lambda}) G(k, t)}{\alpha^{-} \xi_{n}^{p^{-}} / T^{p^{-}} p^{+}} \\
& \leq \frac{\sum_{k=1}^{T} \sup _{|t| \leq \xi_{n}} F(k, t)}{\alpha^{-} \xi_{n}^{p^{-}} / T^{p^{-}} p^{+}} \\
& +\frac{\bar{\mu}}{\bar{\lambda}} \frac{\sum_{k=1}^{T} \sup _{|t| \leq \xi_{n}} G(k, t)}{\alpha^{-} \xi_{n}^{p^{-}} / T^{p^{-}} p^{+}} .
\end{aligned}
$$


Moreover, it follows from Assumption (A1) that

$$
\liminf _{\xi \rightarrow+\infty} \frac{\sum_{k=1}^{T} \sup _{|t| \leq \xi} F(k, t)}{\xi_{n}^{p^{-}}}<+\infty
$$

which concludes that

$$
\lim _{n \rightarrow \infty} \frac{\sum_{k=1}^{T} \sup _{|t| \leq \xi_{n}} F(k, t)}{\xi_{n}^{p^{-}}}<+\infty
$$

Then, in view of (18) and (30), we have

$$
\begin{aligned}
\lim _{n \rightarrow \infty} \frac{\sum_{k=1}^{T} \sup _{|t| \leq \xi_{n}} F(k, t)}{\xi_{n}^{p^{-}}} \\
\quad+\lim _{n \rightarrow \infty} \frac{\bar{\mu}}{\bar{\lambda}} \frac{\sum_{k=1}^{T} \sup _{|t| \leq \xi_{n}} G(k, t)}{\xi_{n}^{p^{-}}}<+\infty,
\end{aligned}
$$

which follows

$$
\lim _{n \rightarrow \infty} \frac{\sum_{k=1}^{T} \sup _{|x| \leq \xi_{n}} F(k, t)+(\bar{\mu} / \bar{\lambda}) G(k, t)}{\xi_{n}^{p^{-}}}<+\infty .
$$

Therefore,

$$
\begin{aligned}
\gamma & \leq \liminf _{n \rightarrow+\infty} \varphi\left(r_{n}\right) \\
& \leq \lim _{n \rightarrow \infty} \frac{\sum_{k=1}^{T} \sup _{|t| \leq \xi_{n}} F(k, t)+(\bar{\mu} / \bar{\lambda}) G(k, t)}{\alpha^{-} \xi_{n}^{p^{-}} / T^{p^{-}} p^{+}}<+\infty .
\end{aligned}
$$

Since

$$
\begin{aligned}
\frac{\sum_{k=1}^{T} \sup _{|t| \leq \xi_{n}} Q(k, t)}{\xi_{n}^{p^{-}}} \leq & \frac{\sum_{k=1}^{T} \sup _{|t| \leq \xi_{n}} F(k, t)}{\xi_{n}^{p^{-}}} \\
& +\frac{\bar{\mu}}{\bar{\lambda}} \frac{\sum_{k=1}^{T} \sup _{|t| \leq \xi_{n}} G(k, t)}{\xi_{n}^{p^{-}}}
\end{aligned}
$$

taking (18) into account, one has

$$
\begin{aligned}
& \frac{T^{p^{-}} p^{+}}{\alpha^{-}} \liminf _{\xi \rightarrow+\infty} \frac{\sum_{k=1}^{T} \sup _{|t| \leq \xi} Q(k, t)}{\xi p^{-}} \\
& \quad \leq \frac{T^{p^{-}} p^{+}}{\alpha^{-}} \liminf _{\xi \rightarrow+\infty} \frac{\sum_{k=1}^{T} \sup _{|t| \leq \xi} F(k, t)}{\xi^{-}}+\frac{\bar{\mu}}{\bar{\lambda}} g_{\infty} .
\end{aligned}
$$

Moreover, since $G$ is nonnegative, we have

$$
\limsup _{|\xi| \rightarrow+\infty} \frac{\sum_{k=1}^{T} Q(k, \xi)}{\xi p^{+}} \geq \limsup _{|\xi| \rightarrow+\infty} \frac{\sum_{k=1}^{T} F(k, \xi)}{\xi p^{+}} .
$$

Therefore, from (35) and (36) and from Assumption (A1) and (33), one has

$$
\begin{aligned}
\bar{\lambda} & \in] \nu_{1}, v_{2}[ \\
& \subseteq] \frac{2 \alpha^{+}}{p^{-} \lim \sup _{|\xi| \rightarrow+\infty}\left(\sum_{k=1}^{T} Q(k, \xi) / \xi p^{+}\right)}, \\
& \frac{\alpha^{-}}{T p^{-} p^{+} \liminf \inf _{\xi \rightarrow+\infty}\left(\sum_{k=1}^{T} \sup _{|t| \leq \xi} Q(k, t) / \xi p^{-}\right)} \\
& \subseteq] 0, \frac{1}{\gamma}[.
\end{aligned}
$$

For fixed $\bar{\lambda}$, inequality (33) assures that condition (b) of Theorem 1 can be used and either $I_{\bar{\lambda}}$ has a global minimum or there exists a sequence $\left\{u_{n}\right\}$ of solutions of the problem $\left(P_{\lambda}^{f, g}\right)$ such that $\lim _{n \rightarrow \infty}\|u\|=+\infty$.

The other step is to verify that the functional $I_{\bar{\lambda}}$ has no global minimum. Since

$$
\frac{1}{\bar{\lambda}}<\frac{p^{-}}{2 \alpha^{+}} \limsup _{|\xi| \rightarrow+\infty} \frac{\sum_{k=1}^{T} F(k, \xi)}{\xi p^{+}},
$$

we can consider a real sequence $\left\{\gamma_{n}\right\}$ with $\gamma_{n}>1$ for all $n \in \mathbb{N}$ and a positive constant $\tau$ such that $\gamma_{n} \rightarrow+\infty$ as $n \rightarrow \infty$ and

$$
\frac{1}{\bar{\lambda}}<\tau<\frac{p^{-}}{2 \alpha^{+}} \frac{\sum_{k=1}^{T} F\left(k, \gamma_{n}\right)}{\gamma_{n}^{p^{+}}}
$$

for each $n \in \mathbb{N}$ large enough. Thus, we consider a sequence $\left\{w_{n}\right\}$ in $X$ defined by setting

$$
w_{n}(k)= \begin{cases}\gamma_{n} & \text { for } k \in[1, T] \\ 0 & \text { for } k=0, T+1\end{cases}
$$

Thus

$$
\begin{aligned}
\left\|w_{n}\right\|^{2} & =\sum_{k=1}^{T+1}\left|\Delta w_{n}(k-1)\right|^{2}=2 \gamma_{n}^{2}, \\
\frac{2 \alpha^{-}}{p^{+}} \gamma_{n}^{p^{-}} & \leq \Phi\left(w_{n}\right) \leq \frac{2 \alpha^{+}}{p^{-}} \gamma_{n}^{p^{+}} .
\end{aligned}
$$

On the other hand, since $G$ is nonnegative, we observe

$$
\Psi\left(w_{n}\right) \geq \sum_{k=1}^{T} F\left(k, \gamma_{n}\right) .
$$

So, from (39), (42), and (43), we conclude that

$$
\begin{aligned}
I_{\bar{\lambda}}\left(w_{n}\right) & =\Phi\left(w_{n}\right)-\bar{\lambda} \Psi\left(w_{n}\right) \\
& \leq \frac{2 \alpha^{+}}{p^{-}} \gamma_{n}^{p^{+}}-\bar{\lambda} \sum_{k=1}^{T} F\left(k, \gamma_{n}\right) \\
& <\frac{2 \alpha^{+}(1-\bar{\lambda} \tau)}{p^{-}} \gamma_{n}^{p^{+}},
\end{aligned}
$$


for every $n \in \mathbb{N}$ large enough. Hence, the functional $I_{\bar{\lambda}}$ is unbounded from below, and it follows that $I_{\bar{\lambda}}$ has no global minimum. Therefore, Theorem 1 assures that there is a sequence $\left\{u_{n}\right\} \subset X$ of critical points of $I_{\bar{\lambda}}$ such that $\lim _{n \rightarrow \infty} \Phi\left(u_{n}\right)=+\infty$, which from Lemma 3 follows that $\lim _{n \rightarrow \infty}\left\|u_{n}\right\|=+\infty$. Hence, we have the conclusion.

Remark 7. Under the conditions

$$
\begin{aligned}
\liminf _{\xi \rightarrow+\infty} \frac{\sum_{k=1}^{T} \sup _{|t| \leq \xi} F(k, t)}{\xi^{-}} & =0, \\
\limsup _{\xi \rightarrow+\infty} \frac{\sum_{k=1}^{T} F(k, \xi)}{\xi p^{+}} & =\infty,
\end{aligned}
$$

Theorem 6 assures that for every $\lambda>0$ and for each $\mu \in\left[0,1 / g_{\infty}\right.$ [ the problem $\left(P_{\lambda}^{f, g}\right)$ admits infinitely many solutions. Moreover, if $g_{\infty}=0$, the result holds for every $\lambda>0$ and $\mu \geq 0$.

Now, we give an application of Theorem 6 as follows.

Example 8. Let $T=10$, let $p(k)=2+k / 5$ for all $k \in[0,10]$, let $\alpha(k)=1+1 / k$ for all $k \in[1,11]$, and let $a_{n}$ be a sequence defined by

$$
\begin{aligned}
a_{1} & =4, \\
a_{n+1} & =1+10 \sqrt{22} a_{n}^{2} \text { for } n \geq 2
\end{aligned}
$$

and let $b_{n}$ be a sequence such that $b_{1}=4^{4}$ and $b_{n}=a_{n}^{4}-a_{n-1}^{4}$ for all $n \geq 2$. Consider the problem

$$
\begin{aligned}
& -\Delta\left(\left(1+\frac{1}{k}\right)|\Delta u(k-1)|^{(k-1) / 5} \Delta u(k-1)\right) \\
& =\lambda f(k, u(k))+\mu e^{k} u(k), \quad k \in[1,10], \\
& u(0)=u(11)=0,
\end{aligned}
$$

where $f(k, t)=e^{k} h(t)$ for all $(k, t) \in[1,10] \times \mathbb{R}$ with

$$
h(t)=\sum_{n=1}^{\infty} 2 b_{n}\left(1-2\left|t-a_{n}+\frac{1}{2}\right|\right) \chi_{\left[a_{n}-1, a_{n}\right]}(t)
$$

$\forall t \in \mathbb{R}$

where $\chi_{[\alpha, \beta]}$ denotes the characteristic function of the interval $[\alpha, \beta]$. According to the above data we have $p^{-}=2, p^{+}=4$, $\alpha^{-}=12 / 11, \alpha^{+}=2$, and $G(k, t)=e^{k} t^{2} / 2$ for all $(k, t) \in$ $[1,10] \times \mathbb{R}$ and it is easy to verify that $a_{n+1}-1>a_{n}$ and $\int_{a_{n}-1}^{a_{n}} h(t) \mathrm{d} t=b_{n}$ for all $n \in \mathbb{N}$. Then, one has $F\left(k, a_{n}\right)=$ $e^{k} \int_{0}^{a_{n}} h(\xi) \mathrm{d} \xi=a_{n}^{4}$, and it is easy to see that

$$
\begin{aligned}
\liminf _{\xi \rightarrow+\infty} \frac{\sum_{k=1}^{T} \sup _{|t| \leq \xi} F(k, t)}{|\xi|^{p^{-}}} \\
=\lim _{n \rightarrow+\infty} \frac{\sum_{k=1}^{T} e^{k} F\left(a_{n+1}-1\right)}{\left(a_{n+1}-1\right)^{2}}
\end{aligned}
$$

$$
\begin{gathered}
=\frac{1}{2200} \lim _{n \rightarrow \infty} \frac{a_{n}^{4} \sum_{k=1}^{10} e^{k}}{a_{n}^{4}}=\frac{1}{2200} \sum_{k=1}^{10} e^{k}, \\
\limsup _{\xi \rightarrow+\infty} \frac{\sum_{k=1}^{T} F(k, t)}{\xi p^{+}}=\lim _{n \rightarrow+\infty} \frac{\sum_{k=1}^{T} F\left(k, a_{n}\right)}{a_{n}^{4}}=\sum_{k=1}^{10} e^{k} .
\end{gathered}
$$

Hence, using Theorem 6, since

$$
\begin{aligned}
g_{\infty} & :=\frac{T^{p^{-}} p^{+}}{\alpha^{-}} \lim _{\xi \rightarrow+\infty} \frac{\sum_{k=1}^{T} \sup _{|t| \leq \xi} G(k, t)}{\xi p^{-}} \\
& =\frac{800}{3} \lim _{\xi \rightarrow+\infty} \frac{\xi^{2} \sum_{k=1}^{T} e^{k}}{\xi^{2}}=\frac{800 \sum_{k=1}^{10} e^{k}}{3}<+\infty
\end{aligned}
$$

problem (47) for every $\lambda \in] 2 / \sum_{k=1}^{10} e^{k}, 6 / \sum_{k=1}^{10} e^{k}[$ and $\mu \epsilon$ $\left[0,\left(6-\lambda \sum_{k=1}^{10} e^{k}\right) / 1600 \sum_{k=1}^{10} e^{k}\right.$ [ has an unbounded sequence of solutions in the space

$$
E_{10}:=\{u:[0,11] \longrightarrow \mathbb{R}: u(0)=u(11)=0\}
$$

Remark 9. Assumption (A1) in Theorem 6 could be replaced by the following more general condition:

(A2) there exist two sequences $\left\{\theta_{n}\right\}$ and $\left\{\eta_{n}\right\}$ with $\eta_{n}>0$ for every $n \in \mathbb{N}$ and $\left(2 \alpha^{+} / p^{-}\right) \theta_{n}^{p^{+}}<\left(\alpha^{-} / T^{p^{-}} p^{+}\right) \eta_{n}^{p^{-}}$for all $n \in \mathbb{N}$ and $\lim _{n \rightarrow+\infty} \eta_{n}=+\infty$ such that

$$
\begin{aligned}
& \lim _{n \rightarrow+\infty} \frac{\sum_{k=1}^{T} \sup _{|t| \leq \eta_{n}} F(k, t)-\sum_{k=1}^{T} F\left(k, \theta_{n}\right)}{\left(\alpha^{-} / T^{p^{-}} p^{+}\right) \eta_{n}^{p^{-}}-\left(2 \alpha^{+} / p^{-}\right) \theta_{n}^{p^{+}}} \\
& <\frac{p^{-}}{2 \alpha^{+}} \limsup _{|\xi| \rightarrow+\infty} \frac{\sum_{k=1}^{T} F(k, \xi)}{\xi p^{+}} .
\end{aligned}
$$

Indeed, clearly, by choosing $\theta_{n}=0$ for all $n \in \mathbb{N}$, from (A2) we obtain (A1). Moreover, if we assume (A2) instead of (A1) and choose $r_{n}=\alpha^{-} \eta_{n}^{p^{-}} / T^{p^{-}} p^{+}$for all $n \in \mathbb{N}$, applying the same argument in the proof of Theorem 6 , we obtain

$$
\begin{aligned}
& \varphi\left(r_{n}\right) \\
& \quad \leq \frac{\sup _{\left.\left.v \in \Phi^{-1}(]-\infty, r_{n}\right]\right)} \Psi(v)-\sum_{k=1}^{T} F\left(k, w_{n}(k)\right)}{r_{n}-\sum_{k=1}^{T+1}(\alpha(k) / p(k-1))|\Delta u(k-1)|^{p(k-1)}} \\
& \quad \leq \frac{\sum_{k=1}^{T} \sup _{|x| \leq \eta_{n}} F(k, t)-\sum_{k=1}^{T} F\left(k, \theta_{n}\right)}{\left(\alpha^{-} / T^{p^{-}} p^{+}\right) \eta_{n}^{p^{-}}-\left(2 \alpha^{+} / p^{-}\right) \theta_{n}^{p^{+}}}
\end{aligned}
$$

where $w_{n}(t)$ is the same as (40) but $\gamma_{n}$ is replaced by $\theta_{n}$. We have the same conclusion as in Theorem 6 with the interval ]$\lambda_{1}, \lambda_{2}$ [ replaced by the interval 


$$
\begin{aligned}
\Lambda^{\prime} & =] \frac{2 \alpha^{+}}{p^{-} \lim \sup _{|\xi| \rightarrow+\infty}\left(\sum_{k=1}^{T} F(k, \xi) / \xi p^{+}\right)}, \\
& \frac{1}{\lim _{n \rightarrow+\infty}\left(\left(\sum_{k=1}^{T} \sup _{|t| \leq \eta_{n}} F(k, t)-\int_{k=1}^{T} F\left(k, \theta_{n}\right)\right) /\left(\left(\alpha^{-} / T p^{-} p^{+}\right) \eta_{n}^{p^{-}}-\left(2 \alpha^{+} / p^{-}\right) \theta_{n}^{p^{+}}\right)\right)}[.
\end{aligned}
$$

Here, we point out a simple consequence of Theorem 6.

Corollary 10. Assume that

(A3) $\liminf \operatorname{in}_{\xi \rightarrow+\infty}\left(\sum_{k=1}^{T} \sup _{|t| \leq \xi} F(k, t) / \xi^{p^{-}}\right)<\alpha^{-} / T^{p^{-}} p^{+}$;

(A4) $\lim \sup _{\xi \rightarrow+\infty}\left(\sum_{k=1}^{T} F(k, \xi) / \xi^{p^{+}}\right)>2 \alpha^{+} / p^{-}$.

Then, for every arbitrary function $g \in C([1, T] \times \mathbb{R}, \mathbb{R})$ whose $G(k, t)=\int_{0}^{t} g(k, \xi) \mathrm{d} \xi$ for every $(k, t) \in[1, T] \times \mathbb{R}$ is a nonnegative function satisfying condition (18) and for every $\mu \in\left[0, \mu_{g, 1}[\right.$, where

$$
\begin{aligned}
& \mu_{g, 1} \\
& \quad:=\frac{1}{g_{\infty}}\left(1-\frac{T^{p^{-}} p^{+}}{\alpha^{-}} \liminf _{\xi \rightarrow+\infty} \frac{\sum_{k=1}^{T} \sup _{|t| \leq \xi} F(k, t)}{\xi^{-}}\right),
\end{aligned}
$$

the problem

$$
\begin{aligned}
& -\Delta\left(\alpha(k)|\Delta u(k-1)|^{p(k-1)-2} \Delta u(k-1)\right) \\
& \quad=f(k, u(k))+\mu g(k, u(k)), \quad k \in[1, T], \\
& u(0)=u(T+1)=0
\end{aligned}
$$

has an unbounded sequence of solutions.

Remark 11. Theorem 5 is an immediate consequence of Corollary 10.

We here give the following consequence of the main result.

Corollary 12. Let $f_{1} \in C([1, T] \times \mathbb{R}, \mathbb{R})$ and let $F_{1}(k, t)=$ $\int_{0}^{t} f_{1}(k, \xi)$ for all $t \in \mathbb{R}$. Assume that

(A5) $\liminf \operatorname{in}_{\xi \rightarrow+\infty}\left(\sum_{k=1}^{T} \sup _{|t| \leq \xi} F_{1}(k, t) / \xi^{p^{-}}\right)<+\infty$;

(A6) $\lim \sup _{\xi \rightarrow+\infty}\left(\sum_{k=1}^{T} F_{1}(k, \xi) / \xi p^{+}\right)=+\infty$.

Then, for every function $f_{i} \in C([1, T] \times \mathbb{R}, \mathbb{R})$, denoting $F_{i}(k, t)=\int_{0}^{t} f_{i}(k, \xi) \mathrm{d} \xi$ for all $t \in \mathbb{R}$ for $2 \leq i \leq n$, satisfying

$$
\begin{gathered}
\max \left\{\sup _{\xi \in \mathbb{R}} F_{i}(k, \xi) ; 2 \leq i \leq n\right\} \leq 0, \\
\min \left\{\liminf _{\xi \rightarrow+\infty} \frac{F_{i}(k, \xi)}{\xi p^{-}} ; 2 \leq i \leq n\right\}>-\infty,
\end{gathered}
$$

for each

$$
\lambda \in] 0, \frac{\alpha^{-}}{T p^{-} p^{+} \liminf _{\xi \rightarrow+\infty}\left(F_{1}(t, \xi) / \xi p^{-}\right)}[,
$$

for every arbitrary function $g \in C([1, T] \times \mathbb{R}, \mathbb{R})$ whose $G(k, t)=\int_{0}^{t} g(k, \xi) \mathrm{d} \xi$ for every $(k, t) \in[1, T] \times \mathbb{R}$, is a nonnegative function satisfying condition (18) and for every $\mu \in\left[0, \mu_{g, \lambda}[\right.$, where

$$
\begin{aligned}
& \mu_{g, \lambda} \\
& :=\frac{1}{g_{\infty}}\left(1-\frac{\lambda T^{p^{-}} p^{+}}{\alpha^{-}} \liminf _{\xi \rightarrow+\infty} \frac{\sum_{k=1}^{T} \sup _{|t| \leq \xi} F_{1}(k, t)}{\xi p^{-}}\right),
\end{aligned}
$$

the problem

$$
\begin{aligned}
& -\Delta\left(\alpha(k)|\Delta u(k-1)|^{p(k-1)-2} \Delta u(k-1)\right) \\
& =\lambda \sum_{i=1}^{n} f_{i}(k, u(k))+\mu g(k, u(k)), \quad k \in[1, T], \\
& u(0)=u(T+1)=0
\end{aligned}
$$

has an unbounded sequence of solutions.

Proof. Set $F(k, \xi)=\sum_{i=1}^{n} F_{i}(k, \xi)$ for all $\xi \in \mathbb{R}$. Assumption (A6) along with the condition

$$
\min \left\{\liminf _{\xi \rightarrow+\infty} \frac{F_{i}(k, \xi)}{\xi^{-}} ; 2 \leq i \leq n\right\}>-\infty
$$

ensures that

$$
\begin{aligned}
\limsup _{\xi \rightarrow+\infty} \frac{\sum_{k=1}^{T} F(k, \xi)}{\xi p^{+}} & =\limsup _{\xi \rightarrow+\infty} \frac{\sum_{i=1}^{n} \sum_{k=1}^{T} F_{i}(k, \xi)}{\xi^{+}} \\
& =+\infty .
\end{aligned}
$$

Moreover, Assumption (A5), together with the condition

$$
\max \left\{\sup _{\xi \in \mathbb{R}} F_{i}(k, \xi) ; 2 \leq i \leq n\right\} \leq 0,
$$

implies that

$$
\begin{aligned}
& \liminf _{\xi \rightarrow+\infty} \frac{\sum_{k=1}^{T} \sup _{|t| \leq \xi} F(k, t)}{\xi p^{-}} \\
& \quad \leq \liminf _{\xi \rightarrow+\infty} \frac{\sum_{k=1}^{T} \sup _{|t| \leq \xi} F_{1}(k, t)}{\xi^{-}}<+\infty .
\end{aligned}
$$

Hence, the conclusion follows from Theorem 6. 
Here we give the following example to illustrate Corol$\operatorname{lary} 12$.
Example 13. Let $T=100, p(k)=(k+100) / 50$ for all $k \in[0,100]$, and $\alpha(k)=e^{k}$ for all $k \in[1,101]$. Let $f_{1}, f_{2}$ : $[1,100] \times \mathbb{R} \rightarrow \mathbb{R}$ be defined by

$$
\begin{aligned}
& f_{1}(k, t)= \begin{cases}h(k) t^{4}\left(5+10 \sin ^{2}(\ln t)+2 \sin (2 \ln t)\right) & \text { if }(k, t) \in[1,100] \times(0,+\infty), \\
0 & \text { if }(k, t) \in[1,100] \times(-\infty, 0],\end{cases} \\
& f_{2}(k, t)=-l(k) \frac{2 t}{\left(1+t^{2}\right)^{2}},
\end{aligned}
$$

respectively, where $h, l:[1,100] \rightarrow \mathbb{R}$ are two nonnegative continuous functions. Consider the problem

$$
\begin{aligned}
& -\Delta\left(e^{k}|\Delta u(k-1)|^{(k-1) / 50} \Delta u(k-1)\right) \\
& =\lambda\left(f_{1}+f_{2}\right)(k, u(k)), \quad k \in[1,100], \\
& u(0)=u(101)=0 .
\end{aligned}
$$

A direct calculation shows $p^{-}=2, p^{+}=4$, and

$$
\begin{aligned}
& F_{1}(k, t) \\
& = \begin{cases}h(k) t^{5}\left(1+2 \sin ^{2}(\ln t)\right) & \text { if }(k, t) \in[1,100] \times(0,+\infty), \\
0 & \text { if }(k, t) \in[1,100] \times(-\infty, 0],\end{cases} \\
& F_{2}(k, t)=-l(k) \frac{t^{2}}{1+t^{2}} .
\end{aligned}
$$

Put

$$
\begin{aligned}
& a_{m}= \begin{cases}m & \text { if } m \text { is even, } \\
e^{-m \pi} & \text { if } m \text { is odd }\end{cases} \\
& b_{m}=e^{m \pi}
\end{aligned}
$$

for every $m \in \mathbb{N}$.

Then

$$
\begin{gathered}
\lim _{m \rightarrow+\infty} \frac{\sum_{k=1}^{T} \sup _{|t| \leq a_{m}} F_{1}(k, t)}{a_{m}^{2}}= \begin{cases}0 & \text { if } m \text { is odd }, \\
+\infty & \text { if } m \text { is even, }\end{cases} \\
\limsup _{m \rightarrow+\infty} \frac{\sum_{k=1}^{T} F_{1}\left(k, b_{m}\right)}{b_{m}^{4}}=+\infty .
\end{gathered}
$$

So,

$$
\begin{gathered}
\liminf _{\xi \rightarrow+\infty} \frac{\sum_{k=1}^{T} \sup _{|t| \leq \xi} F_{1}(k, t)}{|\xi|^{p^{-}}}=0<+\infty, \\
\limsup _{\xi \rightarrow+\infty} \frac{\sum_{k=1}^{T} F_{1}(k, t)}{|\xi|^{p^{+}}}=+\infty .
\end{gathered}
$$

Moreover,

$$
\begin{aligned}
& \sup _{\xi \in \mathbb{R}} F_{2}(k, \xi)=0, \\
& \liminf _{\xi \rightarrow+\infty} \frac{F_{2}(k, \xi)}{\xi p^{-}}=\liminf _{\xi \rightarrow+\infty} \frac{-l(k) \xi^{2}}{\xi^{2}\left(1+\xi^{2}\right)}=0>-\infty .
\end{aligned}
$$

Hence, all assumptions of Corollary 12 with $\mu=0$ are satisfied. So, for every $\lambda \in(0,+\infty)$, problem (66) has an unbounded sequence of solutions in the space

$$
E_{100}:=\{u:[0,101] \longrightarrow \mathbb{R}: u(0)=u(101)=0\} .
$$

Arguing as in the proof of Theorem 6 but using conclusion (c) of Theorem 1 instead of (b), one establishes the following result.

\section{Theorem 14. Assume that}

(A7) $\liminf \inf _{\xi \rightarrow 0^{+}}\left(\sum_{k=1}^{T} \sup _{|t| \leq \xi} F(k, t) / \xi^{p^{-}}\right)<\left(p^{-} \alpha^{-} /\right.$ $\left.2 \alpha^{+} p^{+} T^{p^{-}}\right) \lim \sup _{\xi \rightarrow 0^{+}}\left(\sum_{k=1}^{T} F(k, \xi) / \xi^{p^{+}}\right)$.

Then, for each $\lambda \in] \lambda_{3}, \lambda_{4}[$, where

$$
\begin{aligned}
& \lambda_{3}:=\frac{2 \alpha^{+}}{p^{-} \lim \sup _{\xi \rightarrow 0^{+}}\left(\sum_{k=1}^{T} F(k, \xi) / \xi p^{+}\right)}, \\
& \lambda_{4}:=\frac{\alpha^{-}}{T p^{-} p^{+} \liminf _{\xi \rightarrow 0^{+}}\left(\sum_{k=1}^{T} \sup _{|t| \leq \xi} F(k, t) / \xi p^{-}\right)},
\end{aligned}
$$

for every arbitrary function $g \in C([1, T] \times \mathbb{R}, \mathbb{R})$ whose $G(k, t)=\int_{0}^{t} g(k, \xi) \mathrm{d} \xi$ for every $(k, t) \in[1, T] \times \mathbb{R}$ is a nonnegative function satisfying the condition

$$
g_{0}:=\frac{T^{p^{-}} p^{+}}{\alpha^{-}} \lim _{\xi \rightarrow 0^{+}} \frac{\sum_{k=1}^{T} \sup _{|t| \leq \xi} G(k, t)}{\xi^{-}}<+\infty
$$

and for every $\mu \in\left[0, \mu_{g, \lambda}[\right.$ where

$$
\begin{aligned}
& \mu_{g, \lambda} \\
& :=\frac{1}{g_{0}}\left(1-\frac{\lambda T^{p^{-}} p^{+}}{\alpha^{-}} \liminf _{\xi \rightarrow 0^{+}} \frac{\sum_{k=1}^{T} \sup _{|t| \leq \xi} F(k, t)}{\xi^{p^{-}}}\right),
\end{aligned}
$$

the problem $\left(P_{\lambda}^{f, g}\right)$ has a sequence of pairwise distinct solutions which strongly converges to 0 in $E$. 
Proof. Fix $\bar{\lambda} \in] \lambda_{3}, \lambda_{4}[$ and let $g$ be the function satisfying condition (74). Since $\bar{\lambda}<\lambda_{2}$, one has $\mu_{g, \bar{\lambda}}>0$. Fix $\bar{\mu} \epsilon$ ] $0, \mu_{g, \bar{\lambda}}\left[\right.$ and set $\nu_{3}:=\lambda_{3}$ and $\nu_{4}:=\lambda_{4} /\left(1+(\bar{\mu} / \bar{\lambda}) \lambda_{4} g_{0}\right)$. If $g_{0}=0$, clearly, $\nu_{3}=\lambda_{3}, v_{4}=\lambda_{4}$, and $\left.\lambda \in\right] v_{3}, v_{4}$ [. If $g_{0} \neq 0$, since $\bar{\mu}<\mu_{g, \bar{\lambda}}$, one has

$$
\frac{\bar{\lambda}}{\lambda_{4}}+\bar{\mu} g_{0}<1
$$

and so

$$
\frac{\lambda_{4}}{1+(\bar{\mu} / \bar{\lambda}) \lambda_{4} g_{0}}>\bar{\lambda}
$$

namely, $\bar{\lambda}<v_{4}$. Hence, recalling that $\bar{\lambda}>\lambda_{3}=v_{3}$, one has $\bar{\lambda} \in] v_{3}, v_{4}[$.

Now, put $Q(k, t)=F(k, t)+(\bar{\mu} / \bar{\lambda}) G(k, t)$ for all $t \in \mathbb{R}$ and $k \in[1, T]$. Since

$$
\begin{aligned}
\frac{\sum_{k=1}^{T} \sup _{|t| \leq \xi} Q(k, t)}{\xi^{p^{-}}} \leq & \frac{\sum_{k=1}^{T} \sup _{|t| \leq \xi} F(k, t)}{\xi p^{-}} \\
& +\frac{\bar{\mu}}{\bar{\lambda}} \frac{\sum_{k=1}^{T} \sup _{|t| \leq \xi} G(k, t)}{\xi^{p^{-}}},
\end{aligned}
$$

taking (74) into account, one has

$$
\begin{aligned}
& \frac{T^{p^{-}} p^{+}}{\alpha^{-}} \liminf _{\xi \rightarrow 0^{+}} \frac{\sum_{k=1}^{T} \sup _{|t| \leq \xi} Q(k, t)}{\xi p^{-}} \\
& \quad \leq \frac{T^{p^{-}} p^{+}}{\alpha^{-}} \liminf _{\xi \rightarrow 0^{+}} \frac{\sum_{k=1}^{T} \sup _{|t| \leq \xi} F(k, t)}{\xi p^{-}}+\frac{\bar{\mu}}{\bar{\lambda}} g_{0} .
\end{aligned}
$$

Moreover, since $G$ is nonnegative, from Assumption (A7), we have

$$
\limsup _{\xi \rightarrow 0^{+}} \frac{\sum_{k=1}^{T} Q(k, \xi)}{\xi p^{+}} \geq \limsup _{\xi \rightarrow 0^{+}} \frac{\sum_{k=1}^{T} F(k, \xi)}{\xi^{p^{+}}} .
$$

Therefore, from (79) and (80), we obtain

$$
\begin{gathered}
\bar{\lambda} \in] v_{3}, v_{4}[\subseteq] \frac{2 \alpha^{+}}{p^{-} \lim \sup _{\xi \rightarrow 0^{+}}\left(\sum_{k=1}^{T} Q(k, \xi) / \xi p^{+}\right)}, \\
\frac{\alpha^{-}}{T^{p^{-}} p^{+} \lim \inf _{\xi \rightarrow 0^{+}}\left(\sum_{k=1}^{T} \sup _{|t| \leq \xi} Q(k, t) / \xi p^{-}\right)}[ \\
\subseteq] \lambda_{3}, \lambda_{4}[.
\end{gathered}
$$

We take $X, \Phi, \Psi$, and $I_{\bar{\lambda}}$ as in the proof of Theorem 6 . We prove that $\delta<+\infty$. For this, let $\left\{\xi_{n}\right\}$ be a sequence of positive numbers such that $\xi_{n} \rightarrow 0^{+}$as $n \rightarrow+\infty$ and

$$
\lim _{n \rightarrow \infty} \frac{\sum_{k=1}^{T} \sup _{|t| \leq \xi_{n}} F(k, t)}{\xi_{n}^{p^{-}}}<+\infty .
$$

Put $r_{n}=\left(\alpha^{-} / T^{p^{-}} p^{+}\right) \xi_{n}^{p^{-}}$for all $n \in \mathbb{N}$. Let us show that the functional $I_{\bar{\lambda}}$ has not a local minimum at zero. For this, let $\left\{\gamma_{n}\right\}$ be a sequence of positive numbers and $\tau>0$ such that $\gamma_{n} \rightarrow 0^{+}$as $n \rightarrow \infty$ and

$$
\frac{1}{\bar{\lambda}}<\tau<\frac{p^{-}}{2 \alpha^{+}} \frac{\sum_{k=1}^{T} F\left(k, \gamma_{n}\right)}{\gamma_{n}^{p^{+}}}
$$

for each $n \in \mathbb{N}$ large enough. Let $\left\{w_{n}\right\}$ be a sequence in $X$ defined by (40). So, owing to (42), (43), and (83), we obtain

$$
\begin{aligned}
I_{\bar{\lambda}}\left(w_{n}\right) & =\Phi\left(w_{n}\right)-\bar{\lambda} \Psi\left(w_{n}\right) \\
& \leq \frac{2 \alpha^{+}}{p^{-}} \gamma_{n}^{p^{+}}-\bar{\lambda} \sum_{k=1}^{T} F\left(k, \gamma_{n}\right) \\
& <\frac{2 \alpha^{+}(1-\bar{\lambda} \tau)}{p^{-}} \gamma_{n}^{p^{+}}<0
\end{aligned}
$$

for every $n \in \mathbb{N}$ large enough. Since $I_{\bar{\lambda}}(0)=0$, that means that 0 is not a local minimum of the functional $I_{\bar{\lambda}}$. Hence, part (c) of Theorem 1 ensures that there exists a sequence $\left\{u_{n}\right\}$ in $X$ of critical points of $I_{\bar{\lambda}}$ such that $\left\|u_{n}\right\| \rightarrow 0$ as $n \rightarrow \infty$, and the proof is complete.

Remark 15. Applying Theorem 14, results similar to Remark 9 and Corollaries 10 and 12 can be obtained.

We end this paper by giving the following example as an application of Theorem 14 .

Example 16. Let $T=5, p(k)=k^{2}+2$ for all $k \in[0,5]$, and $\alpha(k)=k^{2}+1$ for all $k \in[1,6]$ and let $f_{1}:[1, T] \times(\mathbb{R} \backslash\{0\}) \rightarrow \mathbb{R}$ be the function defined by

$$
\begin{gathered}
f_{1}(t)=2 t\left(\ln \left(\ln \left(\frac{1}{t^{2}}\right)\right)-\ln ^{-1}\left(\frac{1}{t^{2}}\right)\right) \\
\cdot \sin ^{2}\left(\ln \left(\ln \left(\ln \left(\frac{1}{t^{2}}\right)\right)\right)\right)-4 t \ln ^{-1}\left(\frac{1}{t^{2}}\right) \\
\cdot \sin \left(\ln \left(\ln \left(\ln \left(\frac{1}{t^{2}}\right)\right)\right)\right)+8 t \ln ^{-2}\left(\frac{1}{t^{2}}\right) \\
\cdot\left(1+\ln \left(\frac{1}{t^{2}}\right)\right), \\
f(k, t)= \begin{cases}e^{k} f_{1}(t) & \text { if }(k, t) \in[1,5] \times(\mathbb{R} \backslash\{0\}), \\
0 & \text { if }(k, t) \in[1,5] \times\{0\} .\end{cases}
\end{gathered}
$$

Let $g(k, t)=t^{2} \ln \left(k^{2}\right)$ for all $(k, t) \in[1,5] \times \mathbb{R}$. Direct calculations show $p^{-}=2, p^{+}=27, \alpha^{-}=2, \alpha^{+}=26$, $G(k, t)=t^{3} \ln \left(k^{2}\right) / 3$ for all $(k, t) \in[1,5] \times \mathbb{R}$, and 


$$
F(k, t)= \begin{cases}e^{k} t^{2} \ln \left(\ln \left(\frac{1}{t^{2}}\right)\right) \sin ^{2}\left(\ln \left(\ln \left(\ln \left(\frac{1}{t^{2}}\right)\right)\right)\right)+4 t^{2} \ln ^{-1}\left(\frac{1}{t^{2}}\right), & \text { if }(k, t) \in[1, T] \times(\mathbb{R} \backslash\{0\}) \\ 0 & \text { if }(k, t) \in[1, T] \times\{0\}\end{cases}
$$

Thus

$$
\begin{aligned}
& \liminf _{\xi \rightarrow 0^{+}} \frac{\sum_{k=1}^{T} \sup _{|t| \leq \xi} F(k, t)}{\xi p^{-}} \\
& \quad=\liminf _{\xi \rightarrow 0^{+}} \frac{\sum_{k=1}^{5} \sup _{|t| \leq \xi} F(k, t)}{\xi^{2}}=0, \\
& \limsup _{\xi \rightarrow 0^{+}} \frac{\sum_{k=1}^{T} F(k, \xi)}{\xi p^{+}}=\underset{\xi \rightarrow 0^{+}}{\limsup } \frac{\sum_{k=1}^{5} F(k, \xi)}{\xi^{27}}=+\infty .
\end{aligned}
$$

Hence, using Theorem 14, since

$$
g_{0}:=\frac{T^{p^{-}} p^{+}}{\alpha^{-}} \lim _{\xi \rightarrow 0^{+}} \frac{\sum_{k=1}^{T} \sup _{|t| \leq \xi} G(k, t)}{\xi p^{+}}=0
$$

the problem

$$
\begin{aligned}
& -\Delta\left(\left(k^{2}+1\right)|\Delta u(k-1)|^{k^{2}} \Delta u(k-1)\right) \\
& =\lambda f(k, u(k))+\mu \ln \left(k^{2}\right) u^{2}(k), \quad k \in[1,5], \\
& u(0)=u(6)=0
\end{aligned}
$$

for every $(\lambda, \mu) \in(0,+\infty) \times[0, \infty)$ has a sequence of pairwise distinct solutions which strongly converges to 0 in the space

$$
E_{5}:=\{u:[0,6] \longrightarrow \mathbb{R}: u(0)=u(6)=0\} .
$$

Remark 17. If $f$ and $g$ are nonnegative then the nontrivial solutions of the problem $\left(P_{\lambda}^{f, g}\right)$ are positive. Indeed, let $u_{*}$ be a nontrivial solution of the problem $\left(P_{\lambda}^{f, g}\right)$; assume that the discrete interval $\mathscr{A}=\left\{k \in[1, T] ; u_{*}(k)<0\right\}$ is nonempty. Put $\bar{v}(k)=\min \left\{u_{*}(k), 0\right\}$ for $k \in[1, T]$. Clearly, $\bar{v} \in E$ and one has

$$
\begin{aligned}
& \sum_{k=1}^{T+1} \alpha(k)\left|\Delta u_{*}(k-1)\right|^{p(k-1)-2} \Delta u_{*}(k-1) \Delta \bar{v}(k-1) \\
& \quad-\lambda \sum_{k=1}^{T} f\left(k, u_{*}(k)\right) \bar{v}(k)-\mu \sum_{k=1}^{T} g\left(k, u_{*}(k)\right) \bar{v}(k) \\
& \quad=0 .
\end{aligned}
$$

Since $f$ and $g$ are nonnegative, by choosing $\bar{v}=u_{*}$, one has

$$
0 \leq \alpha^{-} \sum_{k \in \mathscr{A}}\left|\Delta u_{*}(k-1)\right|^{p(k-1)} \leq 0
$$

that is,

$$
\left\|u_{*}\right\|_{\mathscr{A}} \leq 0
$$

which contradicts with the fact that $u_{*}$ is a nontrivial solution. Hence, $u_{*}$ is positive.

\section{Competing Interests}

The authors declare that there are no competing interests regarding the publication of this paper.

\section{References}

[1] W. G. Kelley and A. C. Peterson, Difference Equations: An Introduction with Applications, Academic Press, San Diego, Calif, USA, 1991.

[2] F. M. Atici and A. Cabada, "Existence and uniqueness results for discrete second-order periodic boundary value problems," Computers \& Mathematics with Applications, vol. 45, no. 6-9, pp. 1417-1427, 2003.

[3] F. M. Atici and G. Sh. Guseinov, "Positive periodic solutions for nonlinear difference equations with periodic coefficients," Journal of Mathematical Analysis and Applications, vol. 232, no. 1, pp. 166-182, 1999.

[4] L.-H. Bian, H.-R. Sun, and Q.-G. Zhang, "Solutions for discrete $p$-Laplacian periodic boundary value problems via critical point theory," Journal of Difference Equations and Applications, vol. 18, no. 3, pp. 345-355, 2012.

[5] G. Bonanno and P. Candito, "Nonlinear difference equations investigated via critical point methods," Nonlinear Analysis: Theory, Methods \& Applications, vol. 70, no. 9, pp. 3180-3186, 2009.

[6] A. Cabada, A. Iannizzotto, and S. Tersian, "Multiple solutions for discrete boundary value problems," Journal of Mathematical Analysis and Applications, vol. 356, no. 2, pp. 418-428, 2009.

[7] P. Candito and N. Giovannelli, "Multiple solutions for a discrete boundary value problem involving the $p$-Laplacian," Computers \& Mathematics with Applications, vol. 56, no. 4, pp. 959-964, 2008.

[8] O. Chakrone, E. M. Hssini, M. Rahmani, and O. Darhouche, "Multiplicity results for a $p$-Laplacian discrete problems of Kirchhoff type," Applied Mathematics and Computation, vol. 276, pp. 310-315, 2016.

[9] J. Chu and D. Jiang, "Eigenvalues and discrete boundary value problems for the one-dimensional p-Laplacian," Journal of Mathematical Analysis and Applications, vol. 305, no. 2, pp. 452465, 2005.

[10] S. Heidarkhani, G. Caristi, and A. Salari, "Perturbed Kirchhofftype $p$-Laplacian discrete problems," Collectanea Mathematica, 2016.

[11] S. Heidarkhani and M. Khaleghi Moghadam, "Existence of three solutions for perturbed nonlinear difference equations," Opuscula Mathematica, vol. 34, no. 4, pp. 747-761, 2014.

[12] J. Henderson and H. B. Thompson, "Existence of multiple solutions for second-order discrete boundary value problems," Computers \& Mathematics with Applications, vol. 43, no. 10-11, pp. 1239-1248, 2002.

[13] L. Jiang and Z. Zhou, "Three solutions to Dirichlet boundary value problems for $p$-Laplacian difference equations," Advances 
in Difference Equations, vol. 2008, Article ID 345916, pp. 1-10, 2008.

[14] M. Khaleghi Moghadam and S. Heidarkhani, "Existence of a non-trivial solution for nonlinear difference equations," Differential Equations \& Applications, vol. 6, no. 4, pp. 517-525, 2014.

[15] M. K. Moghadam, S. Heidarkhani, and J. Henderson, "Infinitely many solutions for perturbed difference equations," Journal of Difference Equations and Applications, vol. 20, no. 7, pp. 10551068, 2014.

[16] A. Kristály, M. Mihăilescu, and V. Rădulescu, "Discrete boundary value problems involving oscillatory nonlinearities: small and large solutions," Journal of Difference Equations and Applications, vol. 17, no. 10, pp. 1431-1440, 2011.

[17] H. Liang and P. Weng, "Existence and multiple solutions for a second-order difference boundary value problem via critical point theory," Journal of Mathematical Analysis and Applications, vol. 326, no. 1, pp. 511-520, 2007.

[18] G. M. Bisci and D. Repovš, "Nonlinear algebraic systems with discontinuous terms," Journal of Mathematical Analysis and Applications, vol. 398, no. 2, pp. 846-856, 2013.

[19] G. Molica Bisci and D. Repovš, "On some variational algebraic problems," Advances in Nonlinear Analysis, vol. 2, no. 2, pp. 127146, 2013.

[20] D.-B. Wang and W. Guan, "Three positive solutions of boundary value problems for $p$-Laplacian difference equations," Computers \& Mathematics with Applications, vol. 55, no. 9, pp. 19431949, 2008.

[21] M. Bojowald, H. H. Hernández, and H. A. Morales Técotl, "Perturbative degrees of freedom in loop quantum gravity: anisotropies," Classical and Quantum Gravity, vol. 23, no. 10, pp. 3491-3516, 2006.

[22] E. Eisenriegler, "Anisotropic colloidal particles in critical fluids," The Journal of Chemical Physics, vol. 121, no. 7, pp. 3299-3322, 2004.

[23] J. Garnier, "High-frequency asymptotics for Maxwell's equations in anisotropic media part I: linear geometric and diffractive optics," Journal of Mathematical Physics, vol. 42, no. 4, pp. 1612-1635, 2001.

[24] J. Garnier, "High-frequency asymptotics for Maxwell's equations in anisotropic media-part II: nonlinear propagation and frequency conversion," Journal of Mathematical Physics, vol. 42, pp. 1636-1654, 2001.

[25] M. Bendahmane and K. H. Karlsen, "Renormalized solutions of an anisotropic reaction-diffusion-advection system with $L^{1}$ data," Communications on Pure and Applied Analysis, vol. 5, no. 4, pp. 733-762, 2006.

[26] M. Bendahmane, M. Langlais, and M. Saad, "On some anisotropic reaction-diffusion systems with L1-data modeling the propagation of an epidemic disease," Nonlinear Analysis: Theory, Methods \& Applications, vol. 54, pp. 617-636, 2003.

[27] J. Weickert, Anisotropic Diffusion in Image Processing, Teubner, Stuttgart, Germany, 1998.

[28] I. Fragalà, F. Gazzola, and B. Kawohl, "Existence and nonexistence results for anisotropic quasilinear elliptic equations," Annales de l'Institut Henri Poincare (C) Non Linear Analysis, vol. 21, no. 5, pp. 715-734, 2004.

[29] A. El Hamidi and J. Vétois, "Sharp Sobolev asymptotics for critical anisotropic equations," Archive for Rational Mechanics and Analysis, vol. 192, no. 1, pp. 1-36, 2009.
[30] M. Mihăilescu, P. Pucci, and V. Rădulescu, "Nonhomogeneous boundary value problems in anisotropic Sobolev spaces," Comptes Rendus Mathematique, vol. 345, no. 10, pp. 561-566, 2007.

[31] M. Mihăilescu, P. Pucci, and V. Rădulescu, "Eigenvalue problems for anisotropic quasilinear elliptic equations with variable exponent," Journal of Mathematical Analysis and Applications, vol. 340, no. 1, pp. 687-698, 2008.

[32] M. Galewski and S. Głąb, "On the discrete boundary value problem for anisotropic equation," Journal of Mathematical Analysis and Applications, vol. 386, no. 2, pp. 956-965, 2012.

[33] M. Galewski and R. Wieteska, "Existence and multiplicity of positive solutions for discrete anisotropic equations," Turkish Journal of Mathematics, vol. 38, no. 2, pp. 297-310, 2014.

[34] M. Galewski and R. Wieteska, "On the system of anisotropic discrete BVPs," Journal of Difference Equations and Applications, vol. 19, no. 7, pp. 1065-1081, 2013.

[35] E. M. Hssini, "Multiple solutions for a discrete anisotropic (p1(k); p2(k))- Laplacian equations," Electronic Journal of Differential Equations, vol. 2015, no. 195, pp. 1-10, 2015.

[36] M. Mihăilescu, V. Rădulescu, and S. Tersian, "Eigenvalue problems for anisotropic discrete boundary value problems," Journal of Difference Equations and Applications, vol. 15, no. 6, pp. 557-567, 2009.

[37] G. Molica Bisci and D. Repovš, "On sequences of solutions for discrete anisotropic equations," Expositiones Mathematicae, vol. 32, no. 3, pp. 284-295, 2014.

[38] R. Stegliński, "On sequences of large solutions for discrete anisotropic equations," Electronic Journal of Qualitative Theory of Differential Equations, vol. 25, pp. 1-10, 2015.

[39] G. Bonanno and G. Molica Bisci, "Infinitely many solutions for a boundary value problem with discontinuous nonlinearities," Boundary Value Problems, vol. 2009, Article ID 670675, 20 pages, 2009.

[40] B. Ricceri, "A general variational principle and some of its applications," Journal of Computational and Applied Mathematics, vol. 113, no. 1-2, pp. 401-410, 2000.

[41] G. Bonanno and B. Di Bella, "Infinitely many solutions for a fourth-order elastic beam equation," Nonlinear Differential Equations and Applications, vol. 18, no. 3, pp. 357-368, 2011.

[42] G. Bonanno and G. Molica Bisci, "A remark on perturbed elliptic Neumann problems," Studia Universitatis "Babeş-Bolyai", Mathematica, vol. 55, no. 4, pp. 17-25, 2010.

[43] J. R. Graef, S. Heidarkhani, and L. Kong, "Infinitely many solutions for systems of Sturm-Liouville boundary value problems," Results in Mathematics, vol. 66, no. 3-4, pp. 327-341, 2014.

[44] S. Heidarkhani, "Infinitely many solutions for systems of $\mathrm{n}$ two-point boundary value Kirchhoff-type problems," Annales Polonici Mathematici, vol. 107, no. 2, pp. 133-152, 2013.

[45] S. Heidarkhani, M. Ferrara, and A. Salari, "Infinitely many periodic solutions for a class of perturbed second-order differential equations with impulses," Acta Applicandae Mathematicae, vol. 139, pp. 81-94, 2015.

[46] S. Heidarkhani and M. Karami, "Infinitely many solutions for a class of Dirichlet boundary value problems with impulsive effects," Bulletin Mathématiques de la Société des Sciences Mathématiques de Roumanie. Tome, vol. 58, no. 106, pp. 167-179, 2015. 
[47] G. M. Bisci and P. F. Pizzimenti, "Sequences of weak solutions for non-local elliptic problems with Dirichlet boundary condition," Proceedings of the Edinburgh Mathematical Society. Series II, vol. 57, no. 3, pp. 779-809, 2014.

[48] M. Galewski, S. Glab, and R. Wieteska, "Positive solutions for anisotropic discrete boundary value problems," Electronic Journal of Differential Equations, vol. 2013, no. 32, pp. 1-9, 2013. 


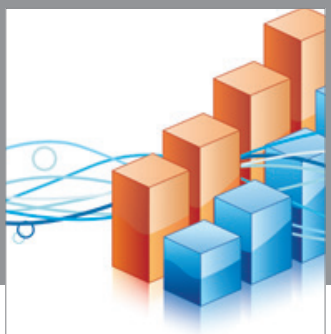

Advances in

Operations Research

vatem alat4

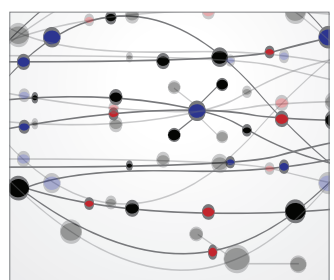

\section{The Scientific} World Journal
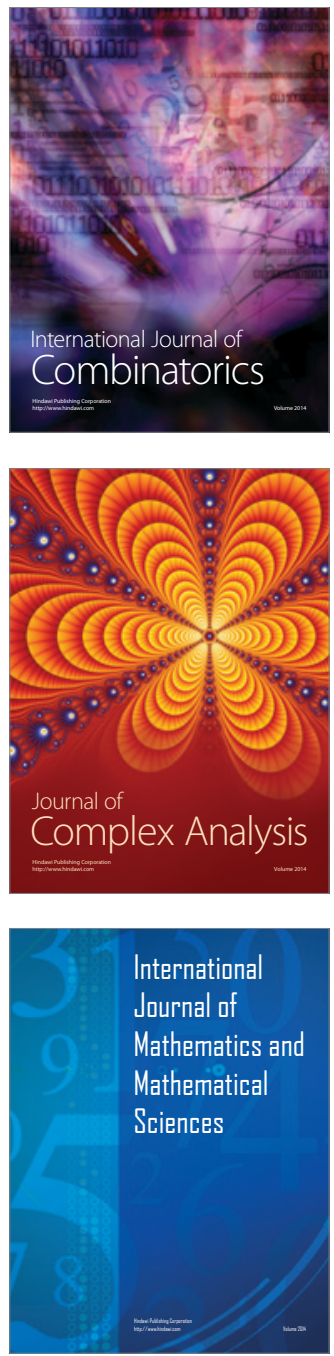
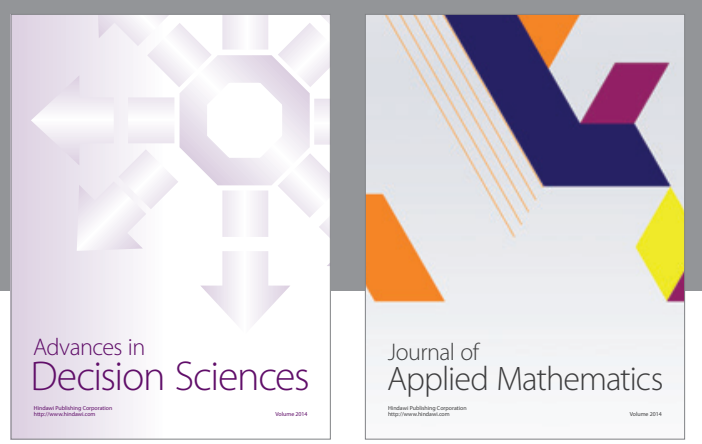

Algebra

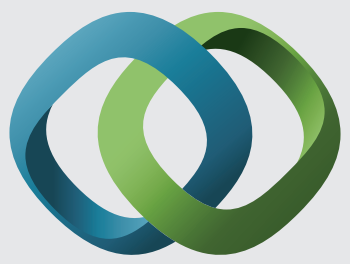

\section{Hindawi}

Submit your manuscripts at

http://www.hindawi.com
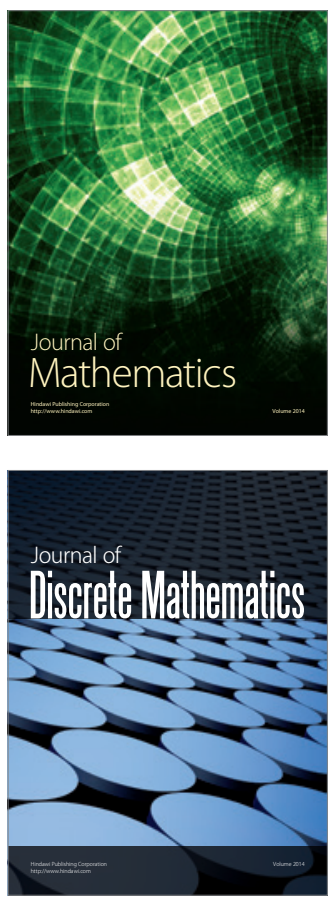

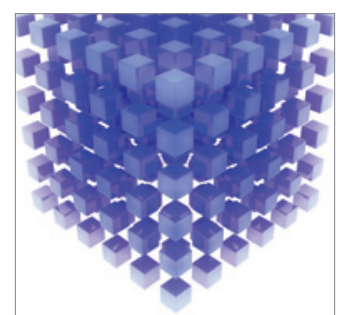

Mathematical Problems in Engineering
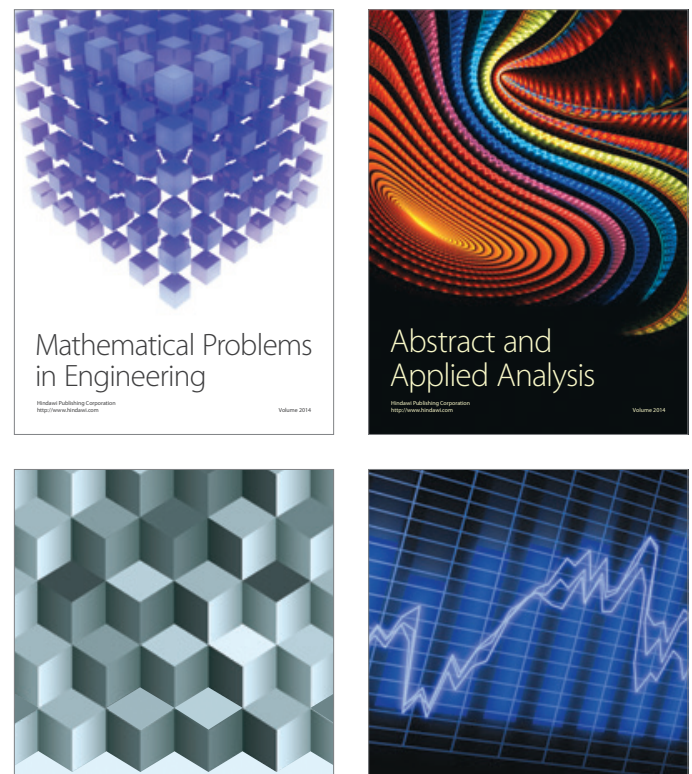

Journal of

Function Spaces

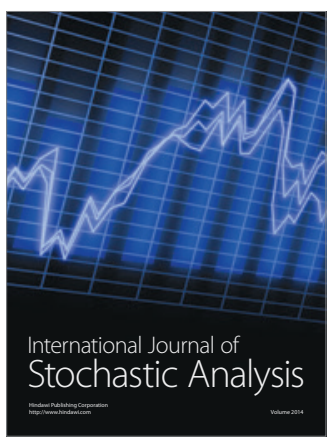

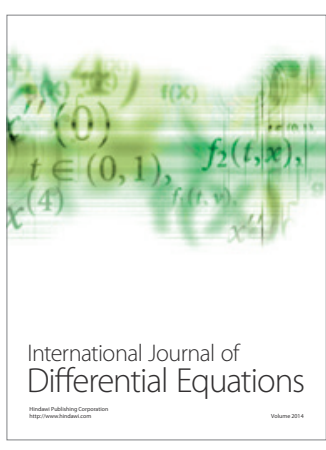
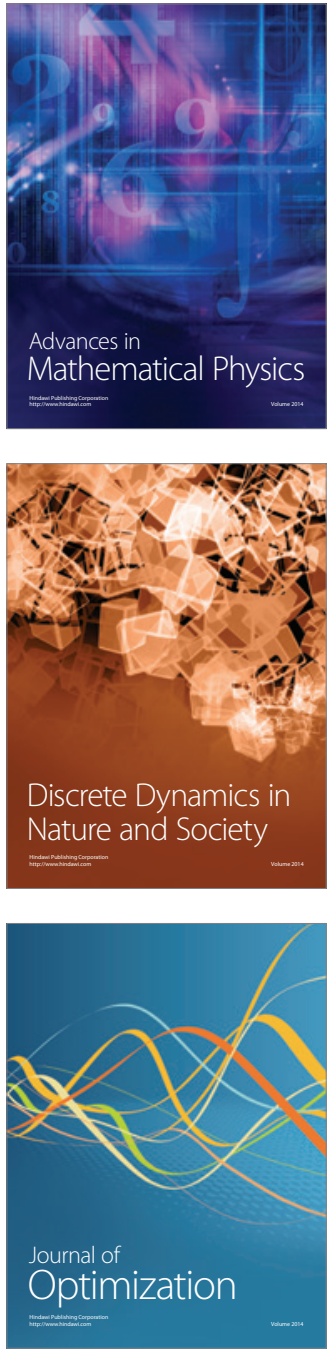